\title{
Shot noise models for sequential processes and the role of lateral mixing
}

\author{
A.R. Neureuther ${ }^{1}$, R.F.W. Pease ${ }^{2}$, L. Yuan ${ }^{3}$, K. Baghbani Parizi ${ }^{2}$, H. Esfandyarpour ${ }^{2}$, \\ W.J. Poppe ${ }^{1}$, J.A. Liddle ${ }^{4}$ and E.H. Anderson ${ }^{4}$ \\ ${ }^{1}$ Department of Electrical Engineering and Computer Sciences \\ University of California- Berkeley, Berkeley, CA 94720 \\ neureuth@eecs.berkeley.edu, tel: (510) 642-4590 fax: (510) 642-2739 \\ ${ }^{2}$ Department of Electrical Engineering, Stanford, 314 CISX \\ ${ }^{3}$ Department of Mechanical Engineering, U.C. Berkeley \\ ${ }^{4}$ Center for X-Ray Optics, LBNL, Berkeley
}

\begin{abstract}
Shot-noise in electron-beam and EUV exposure fundamentally limits the useful sensitivity of resists. Here the exposure, amplification and deprotection of chemically amplified resists are treated as a sequence of statistical events to determine the effect of shot noise. Noise among processes is additive and a sequential Poisson process is used to illustrate the 'acid bottleneck' that occurs when less than one acid electron is generated per input electron. Lateral mixing, due to effects such as electron scattering and acid diffusion, are accounted for in a Binomial sorting model that shows the effect is simply a function of the total number of quanta reaching the voxel irrespective of their path. Electron exposure is estimated to generate on average one acid every 3.8, 21 and $36 \mathrm{~nm}$ along the trajectories of 5, 50 and $100 \mathrm{KeV}$ electrons. Acid generation appears well correlated with energy deposition, at about $22 \mathrm{eV} /$ acid required for EUV and electron exposure, or three times the DUV level. The model was validated by observing the fraction of small ( $24 \mathrm{~nm}$ and $32 \mathrm{~nm}$ across) contacts that cleared as a function of exposure dose at $100 \mathrm{KeV}$. Approximately 4,500 electrons were
\end{abstract}


required to clear, independent of contact size. However, the slope was indicative of a process with only 50 effective events possibly owing to resist surface effects. Circular dissolution smoothing and mechanical fracture are suggested as speculative sources for the near feature size correlation length of the line-edge roughness.

Keywords: Shot Noise, Line Edge Roughness, Line Width Variation, Chemically Amplified Resists, Acid Generation, Poisson, Binomial, Linewidth Variation, Solvent Development, Mechanical Fracture. 


\section{INTRODUCTION}

The local Line-Edge Roughness (LER) and feature scale Line-Width Variation (LWV) of resist profiles are a concern in scaling device dimensions. Patterning results for each new next generation of feature sizes raise concerns until the resist technology matures to address the LER. Yet each step in feature size is one step closer to the underlying fundamental statistical variations in exposure and resist-material response commonly referred to as the shot noise limit. An important materials issue is if the sensitivity improvements needed in Chemically-Amplified Resist Systems (CARS) will cause unacceptable LER. The dependence on the type (photon, electron) or parameters (wavelength, energy) of the exposure is also important. Combining these and other factors in exposure, postexposure bake (PEB), and dissolution is challenging in both the simplest case of developing a contact or in the more complex case of establishing linewidth control.

Shot-Noise effects have been examined from many points of view and a very limited subset of the more recent literature is give here. The recent work by Gallatin [1] is a capstone for modeling many effects. It includes resist dissolution and exposure profile shapes, summarizes recent experimental results and gives algebraic expressions for predicting performance trends. Ocola and collaborators [2-4] have examined electron-beam writing at $100 \mathrm{KeV}$ and made important observations about dose control requirements and the lack of uniformity of energy deposition with distance near the surface of the resist. Their work summarizes the experimental results on dose versus resolution of resists, electron energy deposition mechanisms, resist chemistry, loading and atomistic content and resist dissolution, and folds this work together in Monte Carlo simulation of linewidth control. The recent work of Kruit et al. [5] establishes an algebraic equation that describes the trend with dose, acid diffusion, and device gate length and its calibration via Monte Carlo simulation. Very recently Yu et 
al. [6] reported the possibility of an 'acid generation bottleneck' for electron-beam lithography. Extensive results on the effects of resist variation and the role of image quality have been reported for EUV by Shumway et al. [7] and Cao et al. [8], and for DUV by Palowski et al. [9] and Williamson et al. [10]. Important new experimental work on acid generation has been reported for electron beams by Yamamoto et al. [11] and for EUV by Brainard et al. [12]. SEMs of 30 of sub 50 nm features printed with EUV have been published by Brainard [12] and Shumway et al. [7]. Much earlier Ouano et al. [13] observed the presence of cracks in the dissolution of PMMA. Historically, modeling work has been reported by Everhart et al. [14], Smith [15], and Neureuther et al. [16] and ion beam measurement of Shot Noise were reported by Rau et al. [17]. Further information on modeling, effects of material components, etc. can be found in the references of these authors.

Despite this extensive literature very basic questions remain unanswered. We report in the following sections on four such questions:

1. “Which conditions in electron beam lithography contribute to an 'acid bottleneck' by producing less than one acid per input electron on average during exposure?

2. "How do we combine the variations from different processes that contribute noise?"

3. “Can the presence of an 'acid generation bottleneck' be deduced experimentally from observing the trends in the clearing of arrays of contacts versus exposure dose?”

4. “Are there additional physical phenomena that make LWV larger than that expected from simply averaging LER?

\section{ACID GENERATION}


The results from several research groups can be combined to estimate the rate of generation of acid with electron path length and its relationship to the amount of energy deposited. Yamamoto, et. al [11] characterized the generation of acid in a poly-hydroxyl styrene (PHS) resist with 10\% weight loading of TPS-105 acid generator. A coumarin die was added that was converted in protonated form in the presence of the TPS-105 by the protons created by electron beam exposures. This die was used to verify that the ions generated mostly in the resin were converted to acid generation with essentially no loss. A $75 \mathrm{KeV}$ beam was used and at an exposure dose of $10 \mu \mathrm{C} / \mathrm{cm}^{2}$ an acid concentration of $0.022 \mathrm{acid} / \mathrm{nm}^{3}$ was observed. The acid density can be converted to acids/nm/e by dividing by the electrons $/ \mathrm{cm}^{2}$ in $10 \mu \mathrm{C} / \mathrm{cm}^{2}$ and gives 0.035 acids/nm per electron or one acid per $28.6 \mathrm{~nm}$. Thus for a $25 \mathrm{~nm}$ sized volume element (voxel) at the surface the number of acids is less likely than the number of electrons passing though the volume indicating that an 'acid bottleneck' exists.

As the electron penetrates the resist it slows down and the energy dissipated per unit path length increases according to the Bethe law. For dense exposures the backscattered electrons also contribute to the exposure and under these circumstances simplifying the model derived by Everhart and Hoff [18] to thin layers may be a better approximation. That is, for a thin surface layer (resist) the energy per micron is the incident energy times 0.74 divided by the Gruen range in microns or

$$
\frac{\partial E}{\partial z}=\frac{0.74 E_{\text {BEAM_eV }}}{(0.046 / p) E_{\text {BEAM_KeV }}^{1.75}}=0.76 \mathrm{eV} / \mathrm{nm} \text { for } 75 \mathrm{KeV} \text { incident electron energy }
$$


An important parameter for characterization is the average energy in electron volts to produce one acid. The energy loss in eV/nm divided by the acid/nm gives this ratio which for the above work is 21.7 eV/acid.

A comparison of the energy per acid in eV/acid from several authors is given in Table 1 . Brainard et al. [12] report data on a $30 \mathrm{~nm}$ resolution Shipley EXP EUV resist exposed at both $248 \mathrm{~nm}$ DUV and $13.5 \mathrm{~nm}$ EUV. His values of absorbance and the $125 \mathrm{~nm}$ resist thickness were used to determine the energy per unit volume. Dividing by the $0.032 \mathrm{acid} / \mathrm{nm}^{3}$ that he reports gave the values of 15.2 and $45 \mathrm{eV} / \mathrm{acid}$. Data for the four resists at $248 \mathrm{~nm}$ and $40 \mathrm{KeV}$ was determined from the volumetric dose to clear values from Rau et al. [17] assuming that these doses produced the level of acid used by Brainard et al. or 0.032 acids $/ \mathrm{nm}^{3}$. The value for $100 \mathrm{KeV}$ electron beam exposure is deduced from 0.015 ionizations $/ \mathrm{nm}^{3}$ reported by Ocola et. al [2] for the model resist in a Monte Carlo simulation.

The data in Table 1 show several overall trends. First the EUV and electron-beam values for the same resists are always higher by a factor of about 3 than the DUV values. This is reasonable as less complicated energy transfer mechanisms are needed in DUV. The electron-beam results in the Table and from Yamamoto, et al. are in the range of 20 to 25 eV/acid independent of beam voltage. The EUV value is higher at $45 \mathrm{eV} /$ acid possibly owing to the tuning of this resist to reduce diffusion effects to achieve $30 \mathrm{~nm}$ resolution. The above data suggests that assuming the eV/acid is nearly constant is a useful way to estimate the number of acids created per unit volume as a function of changes in parameters such resist absorbance and electron beam voltage. For example, for 21.7 
eV/acid, one acid is generated on average every 3.8, 21 and $36 \mathrm{~nm}$ along the trajectories of 5, 50 and $100 \mathrm{KeV}$ electrons.

\section{Models for Sequential and Parallel Processes}

The many physical mechanisms that contribute to LER and their statistical models will continue to evolve with further investigation of the physical mechanisms. However, for purposes of illustration here we list mechanisms as either Poisson or Gaussian and assume that they have a single statistic associated with them of their average number $\lambda$ or their standard deviation $\sigma$.

- Source generation of photons or electrons (Poisson $\lambda_{\mathrm{S}}$ )

- $\quad$ Beam spot size (Gaussian, $\sigma_{\mathrm{B}}$ )

- Lateral scattering in resist (Gaussian, $\sigma_{\mathrm{R}}$ )

- Lateral movement of ions (Gaussian, $\sigma_{\mathrm{I}}$ )

- Energy deposition per unit length (Poisson $\lambda_{E}$ )

- Lateral energy spread (Gaussian, $\sigma_{\mathrm{E}}$ )

- Energy conversion to acid (Poisson $\lambda_{\mathrm{C}}$ )

- Acid diffusion (Gaussian, $\sigma_{\mathrm{A}}$ )

- Deprotection per acid (Poisson $\lambda_{\mathrm{D}}$ )

- Dissolution Percolation (Poisson $\lambda_{\mathrm{P}}$ )

- Dissolution Globulation (Gaussian, $\sigma_{\mathrm{G}}$ )

To examine the feasibility of combining noise contributions from multiple steps such as these, we consider three processes in series: electrons arrive, they create acids which in turn create 
deprotection events. A Poisson distribution is assumed for each process. Thus electrons arrive at each voxel with a mean of $N s=\lambda_{s}$; the variance is also given by $\lambda_{s}$. The acid generation process for each electron has Poisson statistic $\lambda_{A}$ and results in the number of acids $N_{A}=\lambda_{S} \lambda_{A}$. The deprotection process for each acid has statistic $\lambda_{D}$ and results in the number of deprotection events $N_{D}=\lambda_{S} \lambda_{A} \lambda_{D}$. As a typical example we assume $\lambda_{S}=512, \lambda_{A}=1 / 8$ and $\lambda_{D}=25$. That is on average 512 electrons create 64 acids that in turn create 1,600 deprotection events.

Exposure shot noise is often estimated by using the square root of the average number of expected exposure events per unit volume. This, however, is not correct when the exposure events result from a cascade of dependent sequential events. Since the second and third events depend on the initial event the standard deviation normalized to the average is not $1 / \sqrt{1,600}=0.025$ but rather on the order of that for the event in least supply (acid) $1 / \sqrt{64}=0.125$. In fact, since the sequential processes continue to add their noise it is actually a bit larger at 0.135 (see below). An analogous example from Oatley et al. [19] is noise in the scanning electron microscope which is the result of the combination of shot noise on the primary beam and the randomness in the generation and detection of secondary electrons.

To formally investigate the combined noise from sequential processes we use Moment Generating Functions (MGF) as described in Grimmett and Stirzaker [20]. The use of the MGF is algebraically efficient and to leads to quite general results. The MGF is defined as a sum of the probability distribution with the nth term weighted by $\mathrm{s}^{\mathrm{n}}$. \{ Note: The authors will likely modify this manuscript to use the more common alternative of the MGF based on $\mathrm{e}^{\mathrm{st}}$. For many distributions this sum can 
be found in closed form. All of the statistical moments can then be found by taking derivatives of MGF with respect to s and evaluating at $s=1$. For example,

$$
A V E=G^{\prime}(s)_{s=1} \quad \operatorname{VAR}=G^{\prime \prime}(s)_{s=1}+G^{\prime}(s)_{s=1}-\left(G^{\prime}(s)_{s=1}\right)^{2}
$$

An interesting property is that a sum of independent identically distributed random variables

$$
S=X_{1}+X_{2}+\ldots+N_{N}
$$

with a common MGF $G_{X}(s)$, where $N$ is a random variable which is independent of the $X_{i}$ and has a $\mathrm{MGF} \mathrm{G}_{\mathrm{N}}(\mathrm{s})$, has a composite MGF given by

$$
G_{S}(s)=G_{N}\left(G_{X}(s)\right)
$$

Here, for example Xi might be the number of deprotection events produced by each of $\mathrm{N}$ acids, or $\mathrm{X}_{\mathrm{i}}$ might be the number of acids produced by by each of $\mathrm{N}$ electrons. The $\mathrm{X}_{\mathrm{i}}$ might also be the success with probability p of passage of an electron into a given voxel where $\mathrm{p}$ is derived from the lateral spreading, etc. For any combination of these sequential processes the MGF is

$$
G_{\text {COMPOSITE }}(s)=G_{1 s t}\left(G_{2 n d}\left(G_{\ldots}\left(G_{\text {Nth }}(s)\right)\right)\right)
$$

As an example, we consider the three sequential Poisson processes of emission, acid generation and deprotection. According to the nesting equation above the MGF for deprotection is

$$
G_{\text {TOTAL_DEPROTECT }}(s)=G_{E}\left(G_{A E}\left(G_{D A}(s)\right)\right)=e^{\lambda_{1}\left(e^{\lambda_{2}\left(e^{\lambda_{3}(s-1)}\right)}\right)}
$$

Taking the first and second derivative and applying the definitions of AVE and VAR above, we compute the noise level that is defined as the standard deviation divided by the average. 


$$
\frac{\sqrt{V A R}}{A V E}=\frac{\sqrt{1+\lambda_{3}+\lambda_{2} \lambda_{3}}}{\sqrt{\lambda_{1} \lambda_{2} \lambda_{3}}}=\sqrt{\frac{1}{\lambda_{1}}+\frac{1}{\lambda_{1} \lambda_{2}}+\frac{1}{\lambda_{1} \lambda_{2} \lambda_{3}}}=\sqrt{\frac{1}{N_{1}}+\frac{1}{N_{2}}+\frac{1}{N_{3}}}
$$

Here $\lambda_{1}, \lambda_{2}$ and $\lambda_{3}$ correspond to $\lambda_{S}, \lambda_{A}$ and $\lambda_{D}$ and $N_{1}, N_{2}$, and $N_{3}$ correspond to $N_{S}, N_{A}$ and $N_{D}$. The latter format shows that at the overall effect can simply be interpreted as a summing up of the variance normalized by the square of the average for each process step.

$$
V A R_{\text {TOT }} / A V E_{\text {TOT }}^{2}=\sum_{\text {Processes }} V A R_{i} / A^{2} E_{i}^{2}
$$

Note that combined effect is dominated by the number of events in least supply. Specifically, for our three step example we find that there is an 'acid-bottleneck' that takes over for an 'electron bottleneck' whenever the parameter for acid generation per electron $\lambda_{2}=\lambda_{A}$ is less unity. This 'acid bottleneck' will still dominate the noise level even when the number of catalytic deprotections per acid is only a few.

A second example, is that of a Poisson process with parameter $\lambda$ followed by a second sorting process in which each output is kept with probability p. This probability p might represent the area under the Gaussian distribution occupied by a voxel. It creates a Binomial distribution for each outcome N of the Poisson process. Putting the MGF for a Binomial into MGF of the Poisson gives

$$
G_{\text {KEEP }}(s)=G_{S}\left(G_{S O R T}(s)\right)=e^{\lambda_{1}(1-p+p s-1)}=e^{\lambda p(s-1)}
$$

The simplification of MGF on the right hand side is a rather surprising but famous result from statistics. Specifically, a Poisson generation followed by binary sorting is identically equal to that of a single Poisson process with a new parameter $\lambda^{\prime}=\mathrm{p} \lambda$. To be clear, the sorting process still adds noise. A quick estimate is to assume that the most likely $\mathrm{N}$ for the Poisson process is $\mathrm{p} \lambda$, and use the 
variance for that Binomial process which is $\lambda p(1-p)$ divided by $(\lambda p)^{2}$. Rigorous analysis shows this intuitive reasoning to be correct. Adding the contributions to the noise level from both steps gives

$$
\sum_{\text {Processes }} V A R_{i} / A_{V E_{i}^{2}}=\frac{\lambda}{\lambda^{2}}+\frac{\lambda p(1-p)}{(\lambda p)^{2}}=\frac{1}{\lambda}+\frac{1-p}{\lambda p}=\frac{1}{\lambda p}
$$

Note that when the Binomial sorting noise contribution adds the exactly correct amount to make the total equal that for a single Poisson process with parameter $\lambda \mathrm{p}<\lambda$.

This sorting process is similar to partition noise in tubes and transistors as considered by Van der Ziel [21]. Whether the analogy is exact is open to question. For example according to van der Ziel's treatment (following that of D. O. North [22]) if the arrival of each electron has no correlation with the arrival of any other electron then the arrival per unit time is described as a Poisson distribution. In a vacuum diode the cathode current is usually space charge limited and hence the arrivals are correlated and exhibit less variance than that indicated by the Poisson distribution. If now a grid is inserted and intercepts individual electrons at random then the resulting values of variance/mean on the plate current and grid currents are increased above that of the cathode current (but still less than that of a Poisson distribution). But if the starting current is Poisson distributed (as for a temperaturelimited cathode) then there is no additional partition noise and both plate current and grid current have values of variance/mean corresponding to a Poisson distribution.

A remaining fundamental question is how the lateral mixing of exposure quanta from different electron-beam spots affects the composite noise level. We consider M Poisson spot exposures each with average $\mathrm{AVE}=\lambda_{\mathrm{S}}$ and $\mathrm{VAR}_{\mathrm{SPOT}}=\lambda_{\mathrm{s}}$. We assume that the beam positions and lateral scattering sorts the $\mathrm{M}$ beams such that the individual probabilities of reaching the exposure voxel of interest are 
$p_{i}(i=1, M)$. Assuming that the exposures are independent, the total variance is the sum of the variances from each beam. This is irrespective of which physical outcome we examine (electrons, acid, deprotection) and the associated number of steps to arrive at that physical outcome. For a combination of the two reasons below the contribution of each spot to the total variance is the variance of a spot exposure without sorting $\mathrm{VAR}_{\mathrm{SPOT}}$ multiplied by the $\mathrm{p}_{\mathrm{i}}$ value for that spot. First, the initial two processes (generation and sorting) combine with variance $\lambda p_{i}$ to scale the electron generation noise component by $\mathrm{p}_{\mathrm{i}}$. Second, all of the variance contributions from the subsequent processes are based on summing over the electrons that entered the voxel or $\lambda \mathrm{p}_{\mathrm{i}}$ and hence scale as well with $\mathrm{p}_{\mathrm{i}}$. The same is, of course true for the contribution to the average from each spot. This there is a factor of the sum over the $\mathrm{p}_{\mathrm{i}}$ 's in the numerator and its square in the denominator. The noise level is thus that for a fully captured spot scaled by the square root of the sum over the $\mathrm{p}_{\mathrm{i}}$ 's.

$$
\sqrt{V A R_{\text {Total }} /\left(A V E_{\text {Total }}\right)^{2}}=\sqrt{\frac{\sum_{\text {Processes }} p_{i} V A R_{\text {spot }}}{\left(\sum_{\text {Processes }} \sum_{i} A V E_{\text {SPOT }}\right)^{2}}}=\sqrt{\frac{V A R_{\text {spot }} \cdot \sum_{\text {Processes }} p_{i}}{A V E_{\text {SPOT }}^{2} \cdot\left(\sum_{\text {Processes }} p_{i}\right)^{2}}}=\frac{\sqrt{\left(V A R_{\text {SPOT }} / A V E_{\text {SPOT }}{ }^{2}\right)^{2}}}{\sqrt{\sum_{\text {Processes }} p_{i}}}
$$

Note that there is no change in the combined noise level other than that associated with the change in the overall number of electrons that reach the voxel. Thus it is likely that all effects of lateral mixing can be accounted for by simply using the number of electrons depositing energy the voxel.

In our case we can treat the arrival of electrons from an adjacent source as if they were intercepted by a grid in the vacuum tube case. Thus it matters not whether the electrons were intended for next door or for the current address; if they started out Poisson distributed that's how they arrive; as long as there is no correlation between arrival events they are Poisson distributed. So for the current 
address all that is relevant is the mean number of electrons per unit area. Lateral spreading of course will have the effect of low pass filtering out high spatial frequency variations but that is a different issue.

\section{EXPERIMENTAL VALIDATION}

To generate possible evidence for an 'acid generation bottleneck' we conducted an experiment on printing arrays of small contacts. We exposed with a beam of $100 \mathrm{KeV}$ electrons at progressively increasing doses in IBM KRS resist. An $8 \mathrm{~nm}$ grid was used with a beam spot size of about $6.5 \mathrm{~nm}$ FWHM. The contact holes were laid out as squares 8, 16, 24, 32 and $40 \mathrm{~nm}$ side length and that contain 1, 4, 9, 16, and 25 beam locations. An array of uniformly sized contacts was exposed at constant dose using 17 rows by 23 columns (391 contacts) that fit the SEM image capture window.

The center-to-center spacing of the holes was much greater than their diameter so that the contribution of backscattered electrons to the exposure should be negligible. Due to this absence of backscatter at $100 \mathrm{KeV}$ the dose to clear is expected to rise from the $30 \mu \mathrm{C} / \mathrm{cm}^{2}$ dose to clear for large areas to $45 \mu \mathrm{C} / \mathrm{cm}^{2}$. Both $40 \mathrm{~nm}$ and $80 \mathrm{~nm}$ resist thicknesses were used and the writing doses ranged to several times the dose to clear. Using the NIH Image software package (http://rsb.info.nih.gov/nih-image/about.html), contacts of an adequate circular size and contrast were automatically identified from SEM micrographs of each full array. The number of printed contacts found was then plotted versus the exposure dose and, as described below, from the shape of this curve we deduce the critical number of exposure quanta.

There was a significant variation in the size and shape of the contacts (Fig. 1) and the location of the contact centroid appears to vary by about as much as $8 \mathrm{~nm}$ in both $\mathrm{x}$ and $\mathrm{y}$. The smallest resolved 
contacts were greater than $20 \mathrm{~nm}$ on a side although the maximum dose was not sufficient to print the 8 and $16 \mathrm{~nm}$ arrays. The dose $\left(\mathrm{C} / \mathrm{cm}^{2}\right)$ to print $50 \%$ of the contacts increased nearly inversely as the areaof the written contact.

More quantitative information can be obtained by plotting the fraction of contacts that printed versus an exposure. If a simple threshold model is used then the fraction of contacts that clear should map out a cumulative Poisson (and approximate a cumulative Gaussian distribution). Data for the cumulative fractions of contacts that opened versus the number of electrons is shown in Fig. 2 for 24 and $36 \mathrm{~nm}$ contacts. Here the number of electrons is shown rather that the dose to reach $50 \%$ printing of the arrays as the former was quite similar at about 4,500 electrons per contact. This trend is consistent with the intrinsic resolution for IBM-KRS resist being $50 \mathrm{~nm}$ and the roughly 1 percent dose control requirement as plotted in Ocola [3] and Liddle et al. [4]. Note also that the resist thickness had little effect.

The major surprise in Figure 2 is that the slope is rather shallow. Numerically the fraction that cleared per electron is between 0.00047 and 0.00060 . The corresponding standard deviation given by $\sigma=1 /(\sqrt{2 \pi})$ slope is 646 to 845 electrons. This gives a noise ratio level $\sigma / \mathrm{AVE}=0.144$ to 0.188. This noise level ratio is equivalent to that of a Poisson distribution with only 28 to 46 events.

The noise level ratio of a sub-50 event process in a 4500 electron process could not be explained by the ‘acid generation bottleneck' even under a worst case assumption that only one out of 10 electrons generated acid. However, the presence of a difficult-to-develop surface layer was observed. An 
example from a $50 \mathrm{~nm}$ line is shown in Figure 3. The surface layer appears to be as thin as a few nm and the developer appears to penetrate in distinct locations that then spread laterally. Ocola [2] has show that the energy deposition at the surface may only be 2/3 of that a few $\mathrm{nm}$ away and in addition acid evaporation or adsorption of a contaminant might occur. The resulting 'surface skin flake’ type resist behavior for early $30 \mathrm{~nm}$ resolution materials can be seen in the EUV exposures of Brainard et al. [12] and Schumway et al. [7]. The use of the above methodology for determining an equivalent effective event count likely be useful in further identifying this issue and testing future materials that address it.

\section{POSSIBLE ADDITIONAL SOURCES OF LINEWIDTH VARIATION}

The polymer resin is also commonly believed to be a major contributor to LER. Many additional factors are possible contributors including phase-separation of dissimilar polymers, the degree of entanglement in the resist and even the basic polymer characteristics. The SEM images in Figures 1 and 3 show a ridged top layer that cantilevers out from the sidewall. The development process appears to start from first opening $10 \mathrm{~nm}$ sized holes in this layer. Then the etching in a circular manner increases several fold in radius while merging between initial openings. As the dissolution process continues the aspect ratios of the remaining roughness at the sidewalls is reduced. This circular dissolution smoothing process appears to be an separate phenomenon from those included in the current models of the LER formation.

Mechanical fracture is another possible but admittedly questionably source of converting fine grain LER to feature sized LWV. SEM images of high resolution features in resist such as shown in Figure 4 suggest even an additional mechanism may be contributing to LWV on a scale larger than that of 
the shot noise mechanisms discussed thus far. Both local scale divots and a much larger gradual change in the linewidth over $150 \mathrm{~nm}$ distances can be seen. Mechanical fracture of silicon wafers after implant as used in wafer bonding technology by Sato et al. [23] shows a surprising similarity to that of this resist sidewall. Quantitatively, even the level of rms roughness, low aspect ratios and large correlation lengths are similar. The ion implant is unnecessary as Drory et al. [24] have shown that layers can be spalled from surfaces simply by depositing and peel thin films. Their experiments and modeling show that depending on the rigidity of the deposited layer a maximum of internal stress occurs within the substrate and at this depth the variation of the sign of the $\mathrm{K}_{\mathrm{II}}$ stress tensor parameter guides the crack propagation toward or away from the interface. During resist development there are mechanical forces within the resist due to the up take of developer. While we usually consider development as making the resist more plastic, Ouano et al. [13] found that in the development of PMMA that this produced cracking perpendicular to the surface. Thus there is also a possibility that during development the resist may remain sufficiently glassy in a thin surface layer also produce lateral spauling like effects. This is depicted in Figure 5 where the exposure and reaction generates polar inhomogeneities, the polar developer penetrates and causes local stress and the fracture follows the path of least resistance. Infrequent 'Lucky Exposure Regions' that occur at $50 \mathrm{~nm}$ or larger separation distances might experience this phenomena first and initiate fracture fronts that enhance the correlation length in LWV from that given by the Fourier transform of the Shot Noise effects.

Any additional polymer related phenomena should express itself in the power spectral density (PSD) of the LER. Looking carefully at the PSD of Cobb in the Gallatin article [1] there is a clear change in slope near 20-30 cycles per micron that might be an indication of the presence of an additional 
physical mechanism. The high frequencies have a downward slope of about 2.5 while the low frequencies have a downward slope of about 1.2. If this is actually indicative of the presence of additional phenomena needs further investigation.

\section{CONCLUSIONS}

Shot-noise in electron-beam and EUV exposure fundamentally limits the useful sensitivity of resists. Here the exposure, amplification and deprotection of chemically amplified resists are treated as a sequence of statistical events to determine the effect of shot noise. This combination of processes can be physically interpreted as contributing noise from the uncertainty in the number of quanta at each step. For example when each electron generates on the average 1 acid then there are contributions to shot noise from both the arrival of electrons and the generation of acids. For Poisson processes the standard deviation due to noise normalized to the average is the squareroot of the sum of one over the quanta of each type. Lateral mixing, due to effects such as electron scattering and acid diffusion, can be accounted for in a Binomial sorting model and shows the effect is simply a function of the total number of quanta reaching the voxel irrespective of their path. During exposure we estimate that on average one acid is generated every 3.8, 21 and $36 \mathrm{~nm}$ along the trajectories of 5, 50 and $100 \mathrm{KeV}$ electrons. Acid generation appears well correlated with energy deposition, at about $22 \mathrm{eV} /$ acid required for EUV and electron exposure, or three times the DUV level. The model was validated by observing the fraction of small (24 nm and $32 \mathrm{~nm}$ diameter) contacts that cleared as a function of exposure dose at $100 \mathrm{KeV}$. Approximately 4,500 electrons were required to clear, independent of contact size. However, the slope was indicative of a process with only 50 effective events possibly owing to resist surface effects. We also suggest that circular dissolution smoothing 
and mechanical fracture are possible although speculative sources for increasing the correlation length of the line-edge roughness.

\section{ACKNOWLEDGEMENTS}

This research was supported in part by SRC under grant SRC 2005-OC-460, DARPA under grant DARPA W911NF-04-1-0304b, industry and the UC Discovery SMART grant ELE03-10160, and by instructional use of the LBNL nanowriter.

\section{REFERENCES}

1) G. M. Gallatin, Proc. SPIE, 5754, 38 (2005).

2) L.E. Ocola, JVST B, 21, 156 (2003).

3) L.E. Ocola, Mat. Res. Soc. Symp. Proc, 705, Y1.1 (2002).

4) J.A. Liddle, G.M. Gallatin, and L.E. Ocola, Mat. Res. Soc. Symp. Proc, 739, H1.5 (2002).

5) P. Kruit, S. Steenbrink, R. Jager, and M. Wieland, JVST. B, 22, 2948 (2004).

6) M.L. Yu, A. Sagle, and B. Buller, Proc. SPIE, 5751, 687 (2001).

7) M.D. Shumway, E.L. Snow, K.A. Goldberg, P. Naulleau, H. Cao, M. Chandhok, J.A. Liddle, E.H. Anderson, and J. Bokor, Proc. SPIE 5374, 454 (2004).

8) A.R. Pawloski, A. Acheta, I. Lalovic, B.M. La Fontaine, and H. J. Levinson, SPIE, 5376, 414 (2004).

9) H.B. Cao, W. Yueh, B.J. Rice, J. Roberts, T. Bacuita, and M. Chandhok, SPIE, 5376, 757 (2004).

10) M. Williamson and A. R. Neureuther, Proc. SPIE, 5039, 423 (2003). 
11) H.Yamamoto, T.Kozawa, "Proton Dynamics in Chemically Amplified Electron Beam Resists”, Japanese Journal of Applied Physics, 43, 848, (2004)

12) R.L.Brainard, P.Trefonas, J.H. Lammers, C.A. Cutler, J.F. Mackevich, A. Trefonas, S.A. Robertson SPIE, 5374, 74 (2004).

13) A.C. Ouano and J.A. Carothers, Polymer Engineering and Science, 20, (1980).

14) T.E. Everhart, I. Sutherland, and C. A. Mead, DARPA Report 1976.

15) H. Smith, J. Vac. Sci. Technol, B, vol. 4, 148 (1986).

16) A.R. Neureuther and C.G. Willson, JVST B, 6, 167 (1986).

17) N. Rau, A. Neureuther,"Sensitivity and image quality of resist with electron-beam, ion-beam, and optical exposure”, SPIE Vol. 3333, 2004, P. 1413-1419.

18) T. E. Everhart, P. H. Hoff, Journal of Applied Physics, 1971, P. 5837- 5846.

19) C. W. Oatley, W. C. Nixon and R. F. W. Pease, Advances in Electronics and Electron Physics, 21, p128 (1965) (Academic press)

20) Grimmett and Stirzaker, 3rd Edition, Oxford, 2001.

21) Van der Ziel A., 'Noise” , p.106-107, (Prentice Hall, 1954).

22) D. O. North, RCA 5, 244 (1941)

23) N. Sato and T. Yonehara, Appl. Phys. Lett. 65, 1924, 1994

24) M.D. Drory, M.D. Thouless, and A.G. Evans, Acta Metall, 36, 2019, (1988). 
Table

Table 1. Energy required to generate one acid under exposure with 248nm photons, $13 \mathrm{~nm}$ photons and electrons exposure and acid (or ionization) density at this energy density. (see text) 


\section{Figures}

Figure 1. SEM of contact holes nominally 40nm square in KRS resist exposed with $100 \mathrm{KeV}$ electrons. The required dose is $70 \mu \mathrm{C} / \mathrm{cm}^{2}$ more than twice that needed to clear large areas.

Figure 2. Plot of fraction of contact holes opened as a function of the average number of exposing electrons/hole. Note that about 4500 electrons are needed independent of resist thickness or hole size in the range 24 to $32 \mathrm{~nm}$ on a side.

Figure 3. SEM of partially developed $50 \mathrm{~nm}$ line showing what appears to be a surface layer acting as a partial barrier to development.

Figure 4. SEM of a developed Shipley UVII-HS resist line illustrating both linewidth variation and line edge roughness (Williamson and Yuan, SPIE 2004)

Figure 5. Model of the effect of fracturing during the development of resist. The exposure and reaction generates polar inhomogeneities, the polar developer penetrates and causes local stress and the fracture follows the path of least resistance. 


\begin{tabular}{|l|c|c|c|c|}
\hline $\begin{array}{l}\text { Exposure } \\
\text { RESIST }\end{array}$ & $\begin{array}{c}\text { DUV } \\
248 \mathrm{~nm}\end{array}$ & $\begin{array}{c}\text { EUV } \\
13.5 \mathrm{~nm}\end{array}$ & $\begin{array}{c}\text { eV/acid } \\
\text { @ KeV }\end{array}$ & acid/nm3 \\
\hline PHS TPS-105 & & & $\begin{array}{c}21.7 \\
@ 75 \mathrm{KeV}\end{array}$ & 0.022 \\
\hline IBM APEX-E & 8.79 & & $\begin{array}{c}19.53 \\
@ 40 \mathrm{KeV}\end{array}$ & 0.032 \\
\hline IBM ENR & 5.86 & & $\begin{array}{c}25.98 \\
@ 40 \mathrm{KeV}\end{array}$ & 0.032 \\
\hline Shipley UVIIHS & 8.79 & & $\begin{array}{c}19.53 \\
@ 40 \mathrm{KeV}\end{array}$ & 0.032 \\
\hline Shipley SAL-601 & & & $\begin{array}{c}25.98 \\
@ 40 \mathrm{KeV}\end{array}$ & 0.032 \\
\hline Shipley EXP & 6.2 & 17.4 & & 0.032 \\
\hline Model Resist & & & $\begin{array}{c}41 \\
@ 100 \mathrm{KeV}\end{array}$ & 0.015 \\
\hline
\end{tabular}

Table 1. Energy required to generate one acid under exposure with 248nm photons, $13 \mathrm{~nm}$ photons and electrons exposure and acid (or ionization) density at this energy density. (see text) 


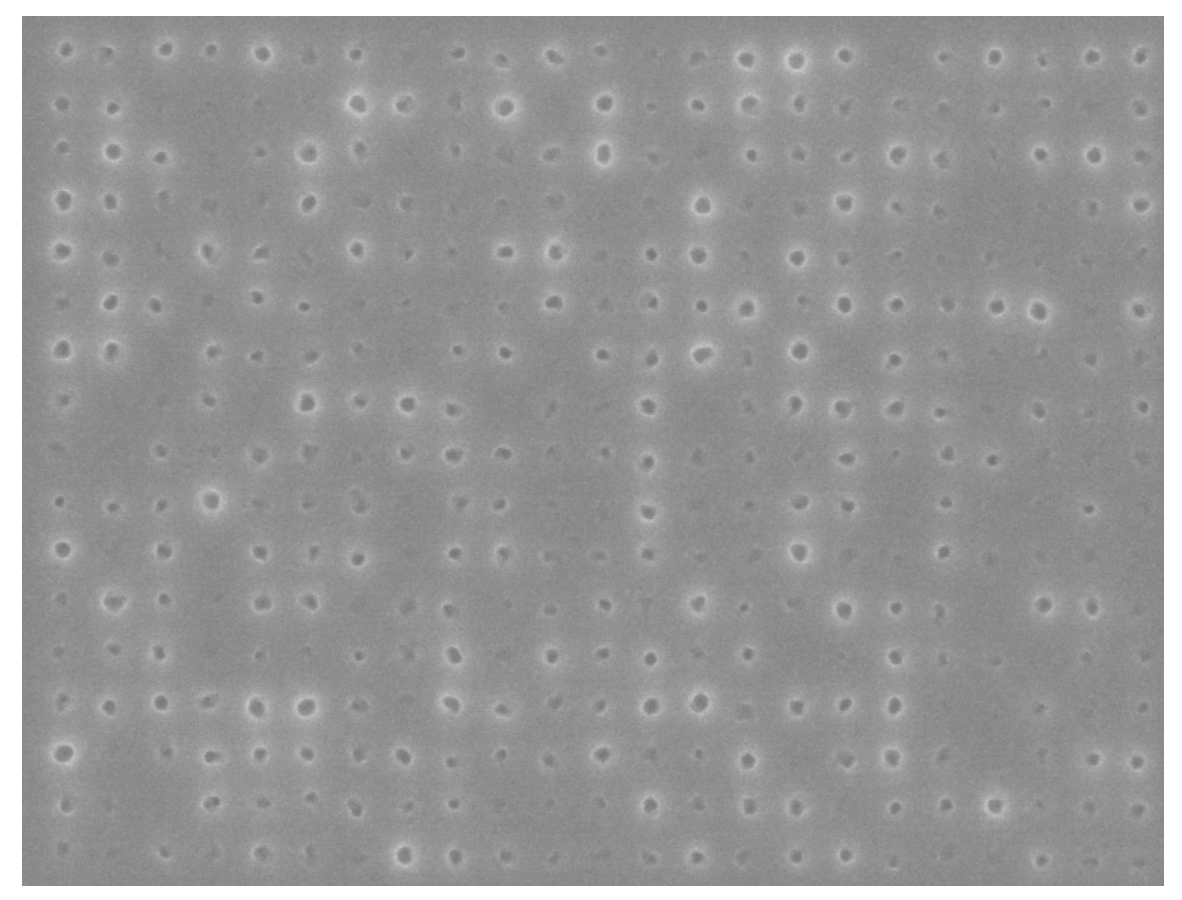

Figure 1. SEM of contact holes nominally 40nm square in KRS resist exposed with $100 \mathrm{KeV}$ electrons. The required dose is $70 \mu \mathrm{C} / \mathrm{cm}^{2}$ more than twice that needed to clear large areas. 




Average number of electrons per feature

Figure 2. Plot of fraction of contact holes opened as a function of the average number of exposing electrons/hole. Note that about 4500 electrons are needed independent of resist thickness or hole size in the range 24 to $32 \mathrm{~nm}$ on a side. 


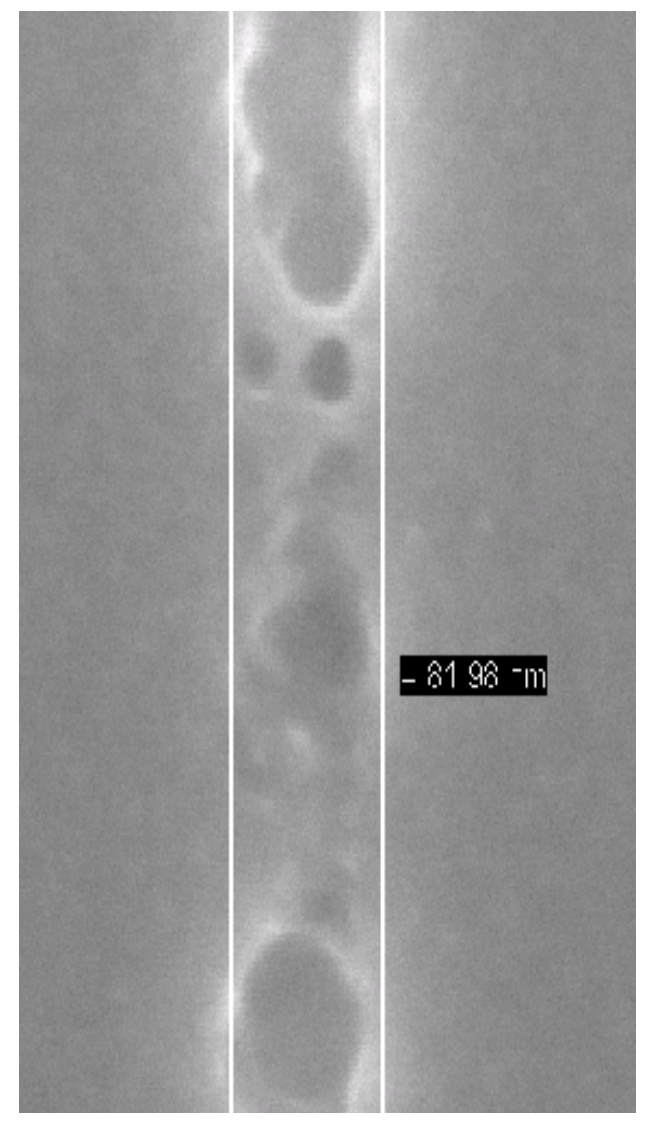

Figure 3 SEM of partially developed line showing what appears to be a surface layer acting as a partial barrier to development. A small variation in the top layer to outgassing could hav a significant effect on dissolution. 


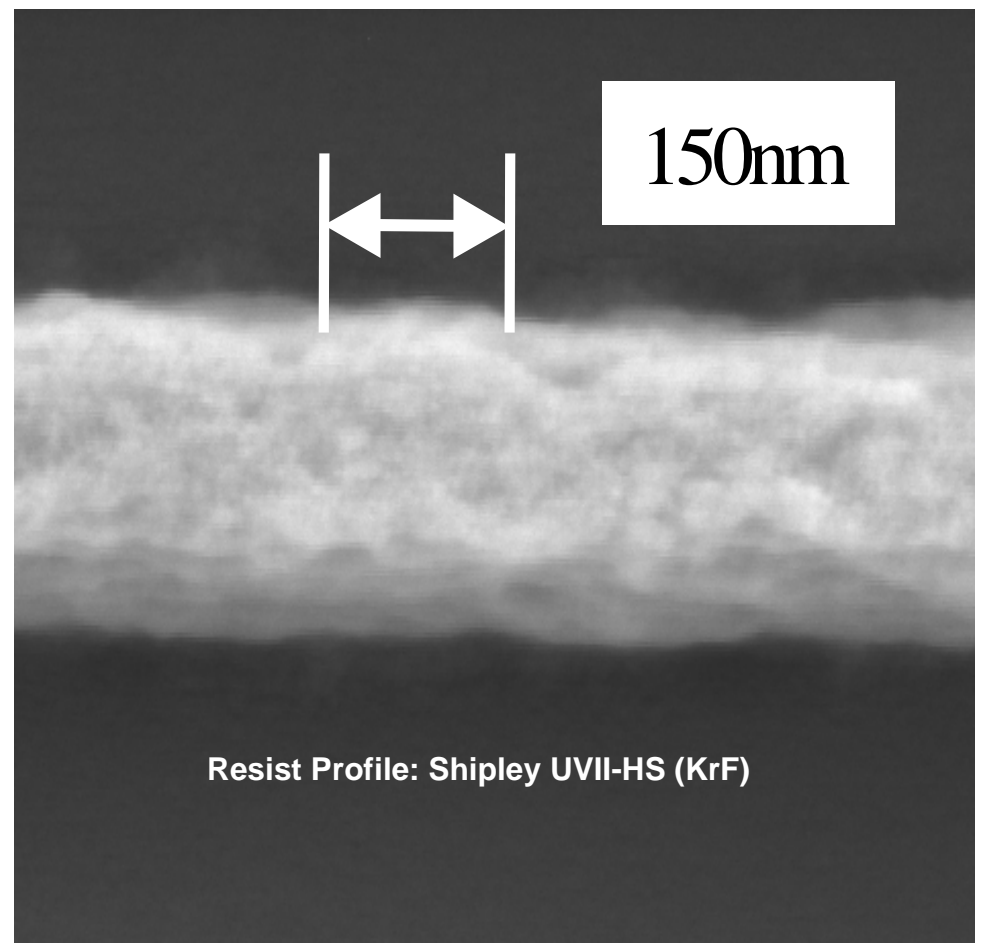

Figure 4. SEM of a developed line illustrating both linewidth variation and line edge roughness.

Note divots and bumps on 30nm scale and gradual linewidth change on 150nm scale (Williamson and Yuan, SPIE 2004). 


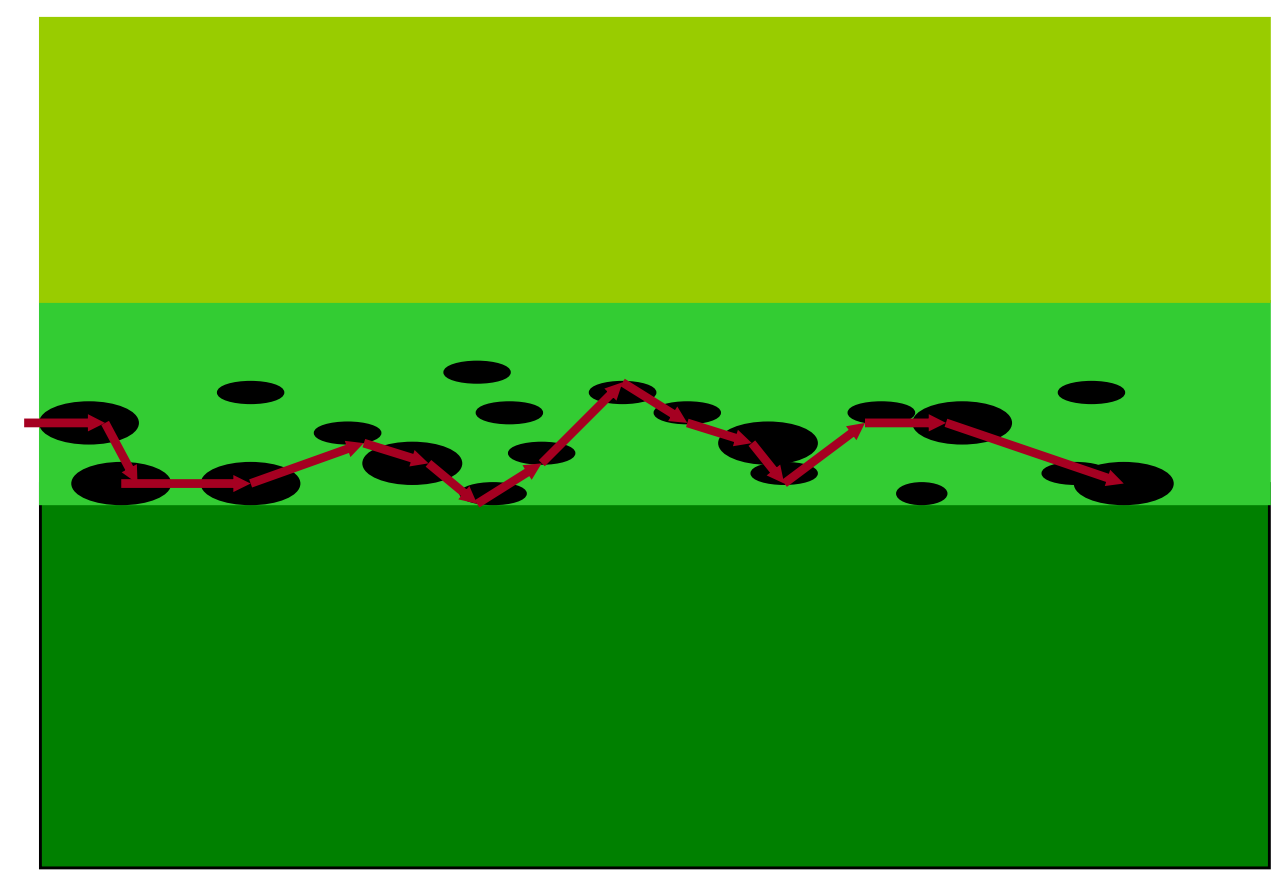

Figure 5. Model of the effect of fracturing during the development of resist. The exposure and reaction generates polar inhomogeneities, the polar developer penetrates and causes local stress and the fracture follows the path of least resistance. 